\title{
Review on Sparse-Based Multipath Estimation and Mitigation: Intense Solution to Counteract the Effects in Software GPS Receivers
}

\author{
Ganapathy Arul Elango, B. Senthil Kumar, \\ Ch.V.M.S.N. Pavan Kumar and C. Venkatramanan
}

Additional information is available at the end of the chapter

http://dx.doi.org/10.5772/intechopen.76521

\begin{abstract}
Multipath is the major concern in GPS receivers that fade the actual GPS signal causes positioning error up to $10 \mathrm{~m}$ so special care need to be taken to mitigate the multipath effects. Numerous methods like hardware based antenna arrays technique, receiver based narrow correlator receiver, double -delta discriminator, Adaptive Multipath Estimator, Wavelet Transformation and Particle filter, Kalman filter based post receiver methods etc. used to resolve the problem. But some of the methods can only reduce code multipath error but not effective in eliminating carrier multipath error. Most of these techniques are based on the assumption that the Line-of-Sight (LOS) signal is stronger than the Non-Line of-Sight (NLOS) signals. However, in the scenarios where the LOS signal is weaker than the composite multipath signal, this approach may result in a bias in code tracking. In this chapter, different types of multipath mitigation and its limitation are described. The recent development in sparse signal processing based blind channel estimation is investigated to compensate the multipath error. The Rayleigh and Rician fading model with different multipath parameters are simulated to test the urban scenario. The inverse problem of finding the GPS signal is addressed based on the deconvolution approach. To solve linear inverse problems, the suitable kind of appropriate objective function has been formulated to find the signal of interest. By exploiting this methods, the signal is observed and the carrier and code tracking loop parameters are computed with minimal error.
\end{abstract}

Keywords: GPS, multipath, Rayleigh, Rician, sparse, de-convolution, ISTA 


\section{Introduction}

Global Positioning System (GPS) is a satellite based navigation system designed and developed by US Department of Defense (DoD) to provide instantaneous 3D position, velocity and time information almost anywhere on or above the surface of the earth at any time, and in any weather. The GPS receiver receives right-hand circularly polarized signals from minimum four satellites to find the user position. The commercial GPS receivers operate at L1 $(1575.42 \mathrm{MHz})$ and L2 (1227.6 MHz) which are modulated on to 50-bps data stream [1]. The positional accuracy provided by GPS is deteriorated by various errors originating at the satellites, Clock error, Ephemeris, Ionospheric, Tropospheric, orbital errors, satellite clock errors, Selective Availability, Receiver Noise and multipath errors. With the use of differential techniques it is possible to remove many of the common-mode error sources, but the error effects of multipath have proven much more difficult to mitigate.

Multipath effect is one of the prominent problems in Wireless communication environment that effects in radio signals reaching the receiving antenna by two or more versions of the transmitted signal arrive at the receiver at slightly different times cause severe degradation in signal reception. Multipath propagation occurs in GPS receivers caused by reflection, refraction, atmospheric ducting, and reflection from nearby objects, water bodies, other reflecting surfaces etc. [2]. The reflecting surface may be buildings, hills, ground, water, or any object that happens to be a radio reflector. The Multipath error result when the receiver receives the direct or line-of-sight (LOS) satellite signal via multiple paths that can be constructively or destructively combined at the receiver antenna to give a resultant signal which can vary widely in amplitude and phase, depending on the distribution of the intensity and relative propagation time of the waves and bandwidth of the transmitted signal. A generic multipath propagation scenario diagram is shown in Figure 1.

\subsection{Overview of GPS signal}

The nominal signal strength of a GPS signal would be around 45-55 dB-Hz. The GPS signal power level lies approximately $15 \mathrm{~dB}$ under the noise background level. The GPS falls in the category of spread spectrum signal having processing gain of $45 \mathrm{~dB}$. By consequence, if an interfering signal is introduced in the receiver with power $45 \mathrm{~dB}$ higher than the noise floor, then the receiver is completely jammed [3]. Interference signals may be in the form of Narrowband and wideband interference. Narrowband can be modeled as continuous wave or pulsed interference at a specified frequency that can be characterized by a pulse duty cycle. Similarly the wideband interference can be modeled as additive white Gaussian noise having flat power spectral density over a wide range of frequencies.

In case, the GPS signal is severely degraded due to Multipath, the signal should be carefully processed by different Multipath algorithms to counteract the effect of diffraction, scattering, Reflection, Refraction, Shadowing etc. The spectrum of the undisturbed (noise free) GPS signal is plotted for a sampling frequency of $5.714 \mathrm{MHz}$ with an IF frequency of $1.6205 \mathrm{MHz}$ in Figure 2. 


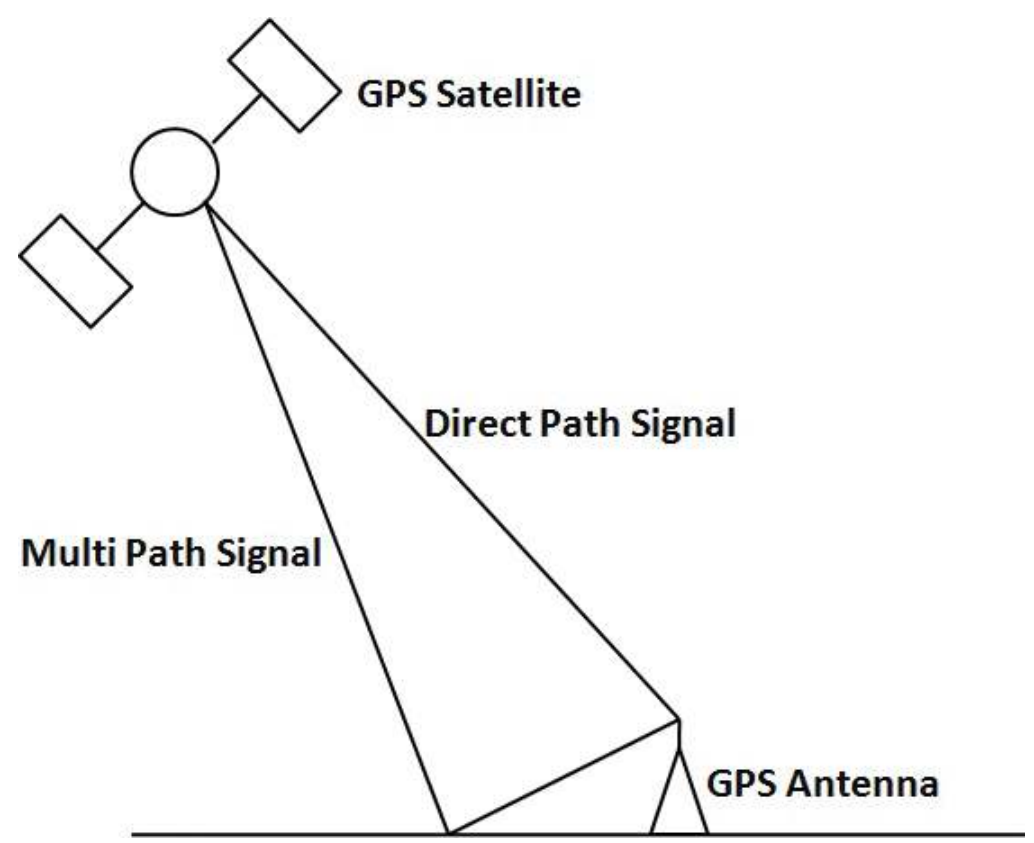

Figure 1. Typical multipath scenario.

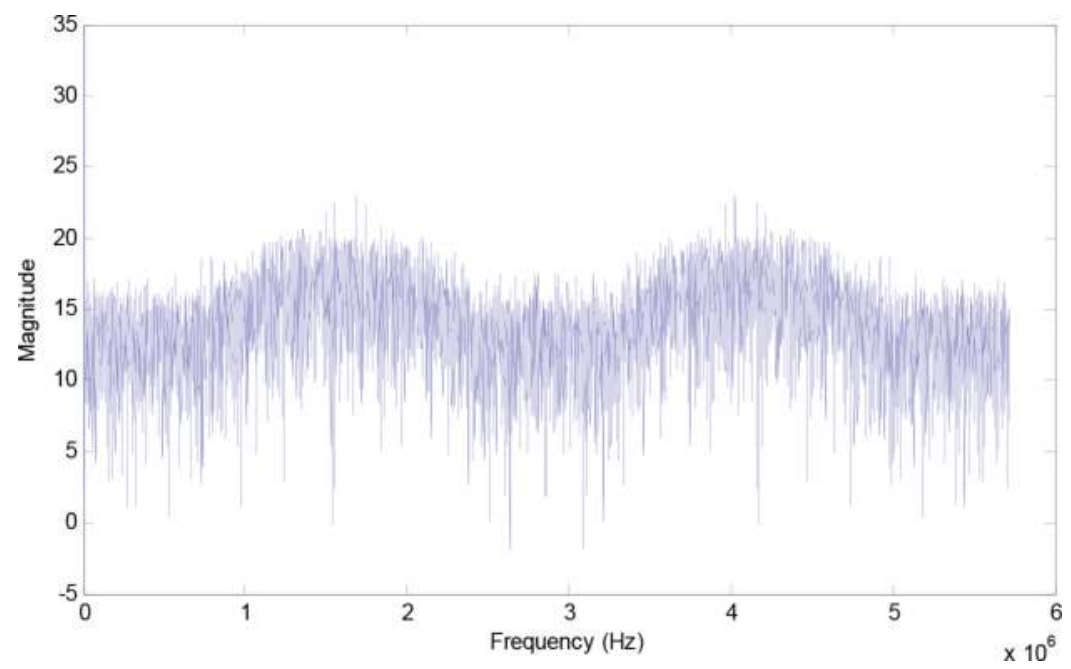

Figure 2. Spectrum of GPS signal with nominal power level. 


\subsection{Overview of multipath channel model}

In mobile (outdoor) radio channels, the Rician distribution is commonly used to describe the statistical time varying nature of the received envelope of a flat fading signal, or the envelope of an individual multipath component. In the channel model, incoming signal is delayed due to different types of obstacles and reached at the receiver side with different time delays with attenuated amplitude and change in phase for each path is shown in Figure 3(a). The complex baseband received signal is given by

$$
y(t)=\sum_{i=0}^{L-1} \alpha_{i} x\left(t-\tau_{i}\right) e^{-j 2 \pi f_{c} \tau_{i}}
$$

$\alpha_{i}$-attenuation in amplitude, $\tau_{\mathbf{i}^{-}}$phase.

As a signal is transmitted, a series of attenuated and delayed versions of the original signal is received leading to a typical multipath channel response. Furthermore, this channel response changes over time.

On the other hand, the indoor propagation channels are characterized by severe multipath propagation. In past two decades, classical Jakes fading model is widely used. In the Jake's Doppler spectrum, the receiver (or transmitter) is assumed to move at certain speed to model the Rayleigh channel. However, in fixed wireless communication systems, both the transmitter and the receiver are stationary and time-variations are actually due to moving scatterers. Filtering White Gaussian Noise (FWGN), AR Model and Sum of Sinusoidal (SOS) exhibits the property of the Rayleigh model [4]. The typical FWGN and AR Model are shown in Figure 3(b) and (c).

From Figure 4(a), the faded envelope is obtained by considering the carrier frequency of $1.6205 \mathrm{MHz}$ and number of multipath components $\mathrm{N}=10$, the Doppler value is kept around

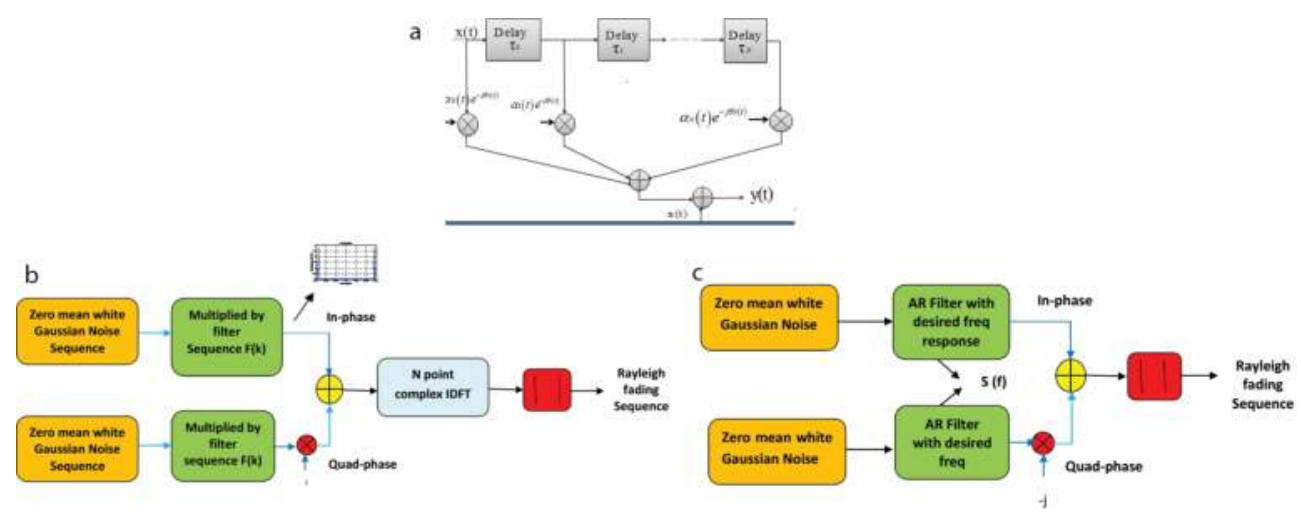

Figure 3. (a) Tapped delay line Multipath Channel model. (b) IDFT model fading simulators. (c) AR model fading simulators. 
(slow moving receiver) $500 \mathrm{~Hz}$ and it is observed that the fading severely degrades the amplitude of the GPS signal and mean variation of the signal is around 0.22 with respect to time when compared to the actual value. To show the effectiveness of the fading the spectrum of the faded signal with SNR of $-15 \mathrm{~dB}$ is plotted in Figure 4(b). A code discriminator in a tracking loop is used to estimate the arrival time of the satellite code. The discriminator function, which is known as the S-curve as shown in Figure $\mathbf{5}$ and it is given by

$$
\mathrm{D}(\tau)=\mathrm{R}_{\mathrm{E}}-\mathrm{R}_{\mathrm{L}}
$$

where $\tau$ is the time of the reference signal. $R_{E}$ and $R_{L}$ are the samples of the early and late correlation functions respectively. The estimated arrival time is the time at which the discriminator is zero. However, in the presence of multipath signals, the autocorrelation function (ACF) will be distorted so that the discriminator will fail to estimate the true arriving time, resulting in pseudo-range estimation error. Due to multipath, the ideal triangular function

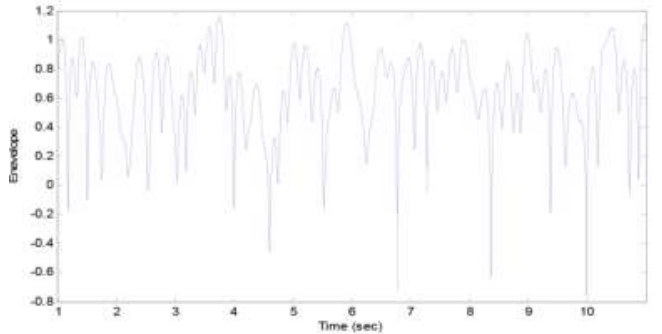

a

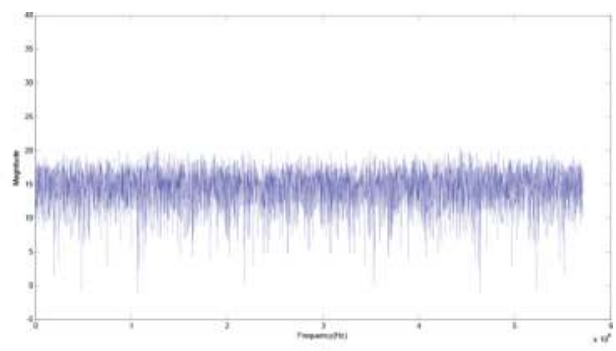

b

Figure 4. (a) Faded envelope. (b) Spectrum of distorted GPS signal with SNR -15 dB.

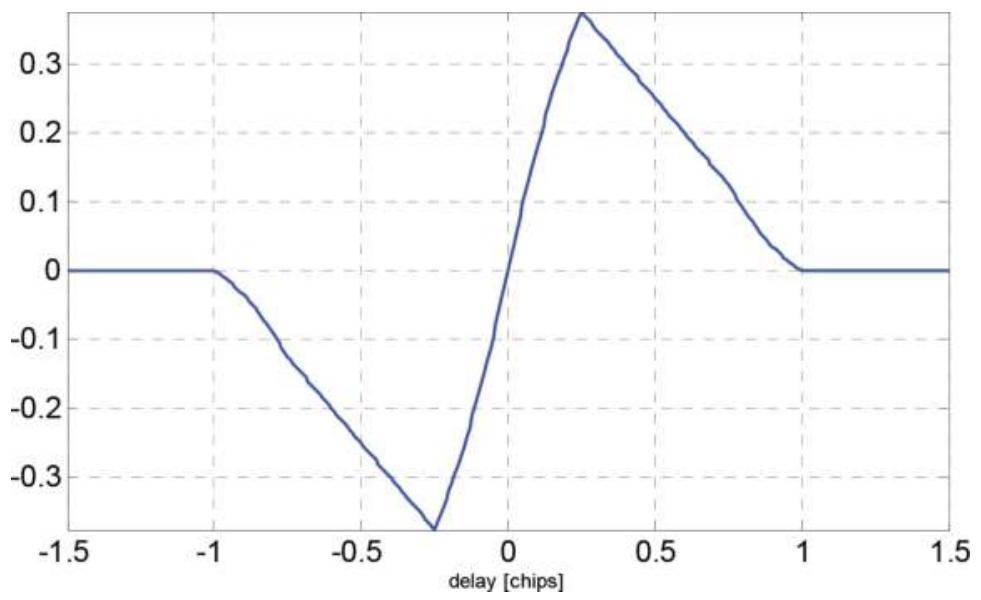

Figure 5. Multipath free discriminator function (s curve) used in tracking stage. 


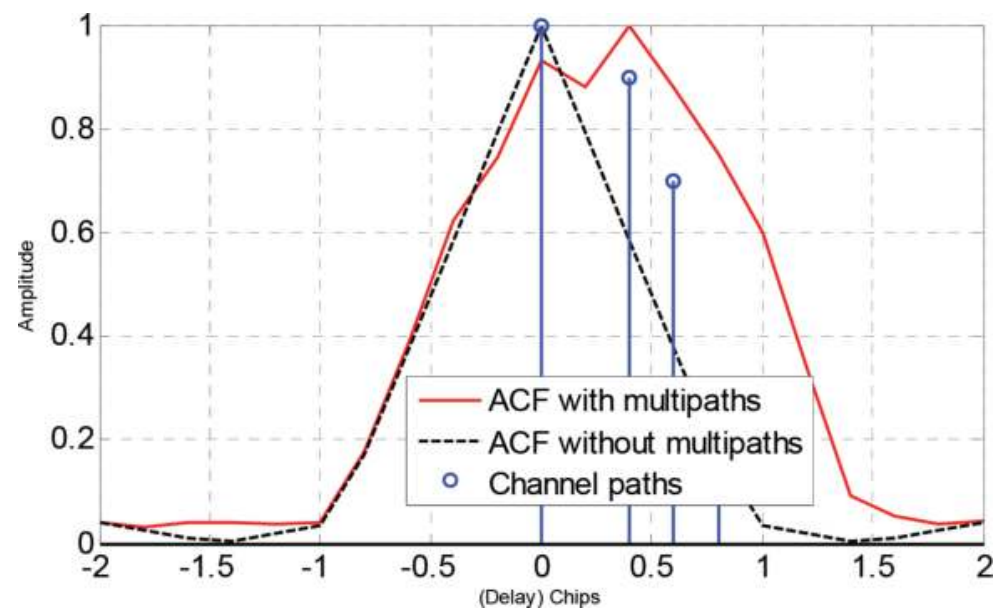

Figure 6. Auto correlation plot with and without multipath component.

loses its symmetry. The distortion of the correlation function is illustrated in Figure 6. When one path is a LOS receives at the receiver which is in phase with the direct signal that follows the ideal triangular shape but in the case of composite 10 path scenario, the correlation function is distorted by 0.4 chips as denoted in the dotted line.

\section{Existing multipath mitigation techniques}

To alleviate multipath problem several pre-filtering and post correlation based methods are introduced. In Many literatures, the problem of multipath is treated inside i.e. signal processing chain of the receiver especially at the stage of tracking and also before signal arrives at the RF front end i.e., at the antenna side, on the other hand some methods describe the effect of multipath is reduced even in the position calculation stage.

\subsection{Miscellaneous methods}

The sparse channel estimation can be estimated by using any one of the estimation techniques like sparse like blind channel estimation or least square based estimation. Once impulse response of the channel is estimated the inverse filter (channel equalizer) is designed to compensate the multipath error. The equalizer output is a delayed version of an impulse response positioned anywhere on the time, finally the LOS signal is observed by subtracting the strongest component from the composite signal. This method combined both estimation and mitigation techniques which is used to compensate the code and carrier tracking error. Some of the other methods deal with mitigation is also given in this section. 


\subsection{Antenna-based mitigation}

Microstrip antennas are frequently used antenna type in GPS receivers because of its added advantage for airborne application, materialization of GPS receiver and easy construction. However, for geodetic needs, antennas are designed to receive both carrier frequencies L1 and L2 [5]. Also they are protected against multipath by extra ground planes or by using choke rings. A choke ring consists of strips of conductor which are concentric with the vertical axis of the antenna and connected to the ground plate which in turns reduces the multipath effect [5]. This involves improving the gain pattern of antenna to counter the effects of multipath. These antenna-based methods include the use of special antennas, processing in spatial domain with multi-antenna arrays, antenna location strategies and long-term signal observation for inferring multipath parameters [6,7]. The circularly polarized antenna facilitates the rejection of multipath signals.

\subsection{Receiver code tracking loop}

The methods include all receiver technologies that are used to mitigate multipath. Usage of Narrow Correlators, Multipath Elimination Technique (MET), Edge Correlator, Strobe Correlator, and Multipath Estimation Delay Lock Loop (MEDLL) and simulation of multipath error in DLL [16] are some of the examples under this category and they will be discussed in detail this section. These techniques, however, are not very effective for short delay multipath [17], due to close-by reflectors. These methods cannot be operated in conjunction with all existing receivers and would need manipulation at the receiver hardware end to work. This remains as one of the major issues with using receiver related techniques [7].

a. Early-minus-late delay lock loop: GPS receiver uses classical correlation-based code tracking structure based on a feedback delay estimator implemented via a feedback loop. The well-known feedback delay estimator is the Early-Minus-Late (EML) DLL, where two correlators spaced one chip apart are used in the receiver in order to form a discriminator function, whose zero crossings determine the path delays of the received signal [8]. The classical EML usually fails to cope with multipath propagation. Therefore, several enhanced EML-based techniques have been introduced in the literature for last two decades in order to mitigate the impact of multipath, especially in closely spaced path scenarios. A first approach to reduce the influences of code multipath is based on the idea of narrowing the spacing between the early and late correlators, i.e., nEML or narrow correlator spacing depends on the receiver's available front-end bandwidth along with the associated sampling frequency.

b. Adaptive Filtering: Multipath Mitigation in GPS/Galileo Receivers with different Signal Processing Techniques has been introduced by Benachenhou et al. [10] efficiently minimize the code and carrier tracking error. Yedukondalu et al. [18] used an adaptive filtering method of estimation and mitigation of Multipath interference in GPS receivers. In this chapter, to estimate the effect of multipath interference at the receiver antenna, a technique based on both code and carrier phase measurements using Code minus Carrier (CMC), is carried out to mitigate multipath for static applications. Different adaptive filters using 
algorithms such as Least Mean Squares (LMS) and various Recursive Least Squares (RLS) are considered to mitigate the error [12]. The estimated multipath error for a typical signal is 0.8 and $2.1 \mathrm{~m}$ on L1 and L2 carriers, respectively.

\subsection{Other filtering methods}

In a simulated multipath environment, the reflection geometry is used in combination with a special GPS antenna arrangement to detect and track multipath. In the highly non stationary environment, Researchers also used Kalman Filter, particle filters and multiple differential GPS receivers to remove multipath errors in final positioning [13]. Code multipath is calibrated and estimated using spherical harmonics in static applications, similarly for kinematic applications, the multipath error mitigation is carried out by Mozaviet et al. [14] using wavelet transform. The estimation of frequency components of multipath error signal using spectral analysis and its effective mitigation using time varying digital filters are designed by Yedukondalu et al. [11]. The four types of filters, namely, Butterworth, Type I and II Chebyshev and Elliptic filters, are examined for mitigation of multipath and their performance are compared. It is observed that by applying digital filters of different cut-off frequencies over the spectrum of the multipath, one can significantly reduce the multipath errors. It was found that Butterworth filter reduced the error most effectively.

\section{Works related to sparse signal processing-based multipath mitigation}

Sparse Signal Processing otherwise known as compressive Sensing (CS), is a classical Signal Processing technique efficiently acquiring and reconstructing a signal completely from reduced number of measurements, by exploiting its compressibility. CS has become a very interesting research area in recent years due to its theoretical and practical utility to capture a wide range of signals at a rate significantly lower than the Nyquist rate representing signal with lesser number of coefficients.

Optimal demodulation and decoding in wireless communication systems often requires accurate knowledge of the channel impulse response. Typically, this is accomplished by searching the channel with a known training sequence and linearly processing the channel with sparse impulse response. On the other hand, conventional linear channel estimation schemes, such as the least-squares method, fail to take advantage of on the anticipated sparsity of the channels. In contrast, it is observed that a CS channel estimate obtained as a solution significantly outperforms a least-squares based channel estimate in terms of the mean squared error (MSE) when it comes to learning sparse (or approximately sparse) channels.

This section highlights some of the most prominent state-of-the-art techniques, which have gained a lot of interest in the research community. The Critical review of literature indicates that exhaustive research has been done by several researchers to develop techniques to improve the performance of software GPS receivers under multipath environment. Researchers have 
concentrated the methods based on compressive sensing implemented in software based GPS receivers for accurate undisturbed reception and positioning.

Dragunas and Borre et al. [19] proposed the sparse deconvolution based Projection onto Convex Sets (POCS) method which is used to mitigate the multipath in indoor environments. The author compared the several multipath mitigation techniques suitable for the indoor environments. By using the proposed method the author chooses one of the secondary paths as LOS signal. In this method, the author achieves better resolution than the conventional methods. An extension to this work, Dragunas et al. [20] presented a modified Projection onto Convex Sets (POCS) that optimizes the Coarse/Acquisition codes employed in Global Positioning Systems. The author deals with the problem of joint LOS code delay and carrier phase estimation of GPS signals in a multipath environment. The modified POCS algorithm acts as the most resistant in closely-spaced multipath static channels both when LOS code delay and carrier phase estimation are concerned. Another sparse based modified iterative Projection onto convex sets (POCS) method proposed by Negin Sokhandan and Ali Broudman [21] is used to reduce the multipath error in harsh environment. The algorithm estimates the channel impulse response (CIR) and removes the spurious noise peaks at each iteration. This method is carried out to estimate the LOS time of arrival from the position of its first non-zero element that passes a certain threshold. The modified POCS algorithm correctly estimates the code delay and carrier phase for GPS signals with few iterations. Hence, faster performance has been achieved when compared to conventional POCS.

Kumar and Lau et al. [22] implemented the deconvolution approach for the code phase and carrier phase estimation. The deconvolution approach shows that it is very different from POCS approach where each path can be estimated. The deconvolution approach can accurately estimate the Line of Sight (LOS) signal. Initially the channel impulse response is computed and by getting the deconvolution filter coefficients, multipath can be removed by convolving the measurements with deconvolution filter coefficients and the code and carrier phase can be estimated and finally the LOS is found.

The novel sparse reconstruction method for mitigating the multipath induced code delay estimation has been implemented by Fei and Liao et al. [23] in GPS receivers. The author exploited to enhance the direct signal without affecting the accuracy of the GPS code delay estimates. The coherent accumulation of received GPS signals and by transforming it into frequency domain and parameters of multipath signals are estimated by sparse reconstruction algorithm. The author estimates the code delay without affecting the accuracy of the GPS by sparse reconstruction method. Tian and Li et al. [24] proposed a novel method based on nonnegative matrix factorization (NMF) spectral unmixing for land seismic additive random noise attenuation. In this method, the noisy seismic signal is first decomposed into a collection of intrinsic mode functions (IMFs) instead of being directly processed. Then, a sparse NMF is used to unmix the STFT spectrum of each IMF. By separating the sub-spectrums by the inverse STFT, the sub-signals can be easily acquired. Finally, the desired signal is reconstructed from the sub-signals by $\mathrm{K}$-means clustering algorithm. Bostan and Kamilov et al. [25] proposed a novel statistically-based discretization paradigm and derive a class of maximum a posterior (MAP) estimators for solving ill-conditioned linear inverse problems. It proposes the theory of 
sparse stochastic processes, which specifies the continuous -domain signals as solutions of linear stochastic differential equations. It provides the algorithm that handles the nonconvex problems and by applying it to the reconstruction algorithm and finally compares the performance of estimators, associated with the models of increasing sparsity.

Broumandan and Lin et al. [15] established a way to enhance the performance of GNSS time arrival estimation techniques in multipath environments by determining the multipath channel estimation using equivalent discrete-time linear time-invariant system method which is modeled as a Moving Average system. It modeled the multipath channel as a sparse channel by describing the number of parameters of the channel is less than the number of unknowns in the Moving Average model. The author compares the performance of the sparse estimation with the Cramer-Rao Lower Bound (CRLB) of the parameter estimation problem and the least square estimate. It provides the better sparse signal recovery method to estimate the channel impulse response.

\subsection{Sparse signal deconvolution}

Sparse de-convolution finds variety of application in accurate estimation of multipath channels with sparse impulse response of a channel is calculated by degradation version of convolution matrix. After down conversion to baseband, the signal from all the satellites can be represented in complex baseband representation as.

$$
y(t)=\sum_{s=0}^{S-1} \alpha, \mathrm{c}_{s}(\mathrm{t}-\tau) \mathrm{e}^{\mathrm{j} 2 \pi\left(\mathrm{f}_{\mathrm{e}}+\mathrm{f}_{\mathrm{d}}\right) t}
$$

where $\alpha^{(\mathrm{s})}$ is the channel attenuation from the $\mathrm{s}^{\text {th }}$ satellite to the receiver, $\tau$ is the time delay or code phase of the C/A code and $f_{d}$ is the Doppler frequency for the $s^{\text {th }}$ satellite.

We assume that the observed GPS signal y from a multipath channel can be written as

$$
y=H x+n
$$

where $\mathrm{x}$ is the signal of interest which is to be estimated, $\mathrm{n}$ is additive noise, and $\mathrm{H}$ is a matrix representing the degradation process. The estimation of actual GPS signal $x$ from the faded version y can be treated as a linear inverse problem. An appropriate objective function, $\mathrm{J}(\mathrm{x})$ has been formulated to solve linear inverse problems and to find the signal $\mathrm{x}$, by minimizing $\mathrm{J}(\mathrm{x})$.

Generally, the chosen objective function is the sum of two terms:

$$
J(x)=D\left(y, H_{x}\right)+\lambda R(x)
$$

where.

$\mathrm{D}\left(\mathrm{y}, \mathrm{H}_{\mathrm{x}}\right)$ measures the discrepancy between $\mathrm{y}$ and $\mathrm{x}$.

$R(x)$ - Regularization term (or penalty function).

$\lambda$-Regularization parameter (positive value). 
To find a signal $x$, so that $H_{x}$ is very similar to $y$, i.e., here it is needed to find a signal $x$ which is consistent with the observed data $\mathrm{y}$. For $\mathrm{D}\left(\mathrm{y}, \mathrm{H}_{\mathrm{x}}\right)$, the mean square error can be calculated as

$$
D\left(y, H_{x}\right)=\left\|y-H_{x}\right\|_{2}^{2}
$$

The squared error between $y$ and $H_{x}$ is minimized by finding the norm difference of $D\left(y, H_{x}\right)$ that will give a signal $x$, which is as consistent with $y$ as possible, according to the square error criterion. To minimize $\mathrm{D}\left(\mathrm{y}, \mathrm{H}_{\mathrm{x}}\right)$ by setting $\mathrm{x}=\mathrm{H}^{-1} \mathrm{y}$; however, $\mathrm{H}$ may not be invertible. Let convolution filter be $\{1,-1,1,-1 \ldots . M\}$ and signal be of length $M$. Convolution sum will have length equal to $\mathrm{N}+\mathrm{M}-1$. So $\mathrm{H}$ in this case will have $\mathrm{N} \times \mathrm{M}-1$ dimension

$$
\mathrm{H}=\left[\begin{array}{lllll}
1 & 0 & 0 & \cdots & 0 \\
-1 & 1 & 0 & \cdots & 0 \\
0 & -1 & 1 & \cdots & \vdots \\
\vdots & 0 & -1 & 1 & 0 \\
0 & \vdots & \cdots & -1 & 1 \\
0 & 0 & \ldots & 0 & -1
\end{array}\right]
$$

which is definitely invertible. Even if $\mathrm{H}$ were invertible, it may be very ill-conditioned, in which case, this solution amplifies the noise, sometimes to such an extent that the solution is useless. The role of the regularization term $R(x)$ is exactly to address this problem. The regularizer $\mathrm{R}(\mathrm{x})$ should be chosen so as to penalize the undesirable/unwanted behavior in $\mathrm{x}$.

\subsubsection{L1-norm regularized linear inverse problem}

By assuming that the GPS signal of interest after acquisition $x$, is known to be sparse. i.e., $x$ has relatively few non-zero values, i.e., $x$ consists of a few impulses and is otherwise zero. In this case, the $R(x)$ may be defined to be the number of non-zero values of $x . R(x)$ is not a convex function of $x$, which is not differentiable then the objective function $J(x)$ is very difficult to minimize and therefore $\mathrm{J}(\mathrm{x})$ will have many local minima. To minimize $\mathrm{J}(\mathrm{x})$, it is better to choose $\mathrm{J}(\mathrm{x})$ to be a convex function of $\mathrm{x}$ that measures sparsity, but which is also convex. For this reason, when $x$ is known to be sparse, the regularization function $R(x)$ is often chosen to be the $\mathrm{L}_{1}$-norm. Hence, the approach is to estimate $\mathrm{x}$ from $\mathrm{y}$ by minimizing the objective function,

$$
\mathrm{J}(\mathrm{x})=\|\mathrm{y}-\mathrm{Hx}\|_{2}^{2}+\lambda\|\mathrm{x}\|_{1}
$$

\subsubsection{Soft-thresholding algorithm (ISTA)}

The requirement for development of fast algorithm is to minimize the equation and its related functions. This is carried out by another significant algorithm called iterated soft-thresholding algorithm (ISTA), also referred as Thresholded Landweber (TL) algorithm. ISTA is a combination of the Landweber algorithm and soft-thresholding. To minimize $\mathrm{J}(\mathrm{x})$, consider first the minimization of the simpler objective function 


$$
\begin{gathered}
J(x)=\|y-H x\|_{2}^{2}=(y-H x)^{T}(y-H x) \\
J(x)=y^{T} y-2 y^{T} H x+x^{T} H^{T} H x
\end{gathered}
$$

Because $\mathrm{J}(\mathrm{x})$ in Eq. (8) is differentiable and convex, thus one can obtain its minimizer by setting the derivative with respect to $x$ to zero. The derivative of $J(x)$ is given by

$$
\frac{\partial}{\partial x} \mathrm{~J}(\mathrm{x})=-2 \mathrm{H}^{\mathrm{T}}+2 \mathrm{H}^{\mathrm{T}} \mathrm{Hx}
$$

Setting the derivative to zero gives a system of linear equations,

$$
\frac{\partial}{\partial x} \mathrm{~J}(\mathrm{x})=0 \text { implies }\left(H^{T} H\right) x=H^{T} y \text {. }
$$

So the minimizer of $\mathrm{J}(\mathrm{x})$ in Eq. (9) is given by

$$
x=\left(H^{T} H\right)^{-1} H^{T} y
$$

\subsubsection{Majorization-minimization (MM) approach}

However, it is not able to solve these equations easily. Since GPS data is a very long, then H will be very large matrix and solving the system of equations may require huge memory and computation time. Moreover, the matrix $\mathrm{H}^{\mathrm{T}} \mathrm{H}$ is not invertible, or ill-conditioned. By using the Majorization-minimization (MM) approach to minimize $J(x)$ in Eq. (10), solving a system of linear equations can be avoided. At each iteration $k$ of the $M M$ approach, a function $G_{k}(x)$ that coincides with $J(x)$ at $x_{k}$ has been found. A majorizer $G_{k}(x)$ has introduced that can be minimized more easily without solving a system of Eqs.

A function $\mathrm{G}_{\mathrm{k}}(\mathrm{x})$ that majorizes $\mathrm{J}(\mathrm{x})$ by adding a non-negative function to $\mathrm{J}(\mathrm{x})$,

$$
G_{k}(x)=J(x)+\text { Non }- \text { negative function of } \mathrm{x}
$$

When $G_{k}(x)$ coincides with $J(x)$ at $x=x_{k}$, the non-negative function added to $J(x)$ should be equal to zero at $x_{k}$ then $G_{k}(x)$ to be

$$
G_{k}(x)=J(x)+(x-x)^{T}\left(\alpha I-H H^{T}\right)\left(x-x_{k}\right)
$$

The function which is added to $J(x)$ is clearly zero at $x_{k}$ so that $G_{k}(x)$ equals to $J\left(x_{k}\right)$ as required. To ensure the function added to $\mathrm{J}(\mathrm{x})$ is non-negative, for all $\mathrm{x}$, the scalar parameter $\alpha$ must be chosen to be equal to or greater than the maximum eigenvalue of $\mathrm{H}^{\mathrm{T}} \mathrm{H}$, i.e., $\alpha \geq \max$ eig $\left(\mathrm{H}^{\mathrm{T}} \mathrm{H}\right)$. Then the matrix $\alpha \mathrm{I}-\mathrm{H}^{\mathrm{T}} \mathrm{H}$ is a positive semi-definite matrix, meaning that $\mathrm{v}^{\mathrm{T}}\left(\alpha \mathrm{I}-\mathrm{H}^{\mathrm{T}} \mathrm{H}\right) \mathrm{v} \geq 0$. Now, using MM procedure, to obtain $x_{k+1}$, function $G_{k}(x)$ is minimized. Expanding $G_{k}(x)$ in (12) gives

$$
G_{k}(x)=y^{T} y-2 y^{T} H x+x^{T} H x+\left(x-x_{k}\right)^{T}\left(\alpha I-H^{T} H\right)\left(x-x_{k}\right)
$$


Note that the quadratic term in Eq. (12) is simply $x^{T} x$ instead of $x^{T} H^{T} H x$. Therefore, we can minimize $G_{k}(x)$ more easily

$$
\begin{gathered}
\frac{\partial}{\partial x} \mathrm{G}_{k}(x)=-2 H^{T} y-2\left(\alpha I-H^{T} H\right) x_{k}+2 \alpha x, \text { Setting, } \frac{\partial}{\partial x} \mathrm{G}_{k}(x)=0 \\
x=x_{k}+\frac{1}{\alpha} H^{T}\left(y-H x_{k}\right)
\end{gathered}
$$

Hence, by using MM procedure to obtain $x$ value at each iteration is given by Landweber update equation as

$$
x_{k+1}=x_{k}+\frac{1}{\alpha} H^{T}\left(y-H x_{k}\right)
$$

\section{Results and discussion}

In this simulation, four multipath components are considered with time varying amplitude and the phase. Initially the GPS signal needs to be framed in the form of sparse signal. This can be done in the acquisition stage only, the sparse representation of this signal easily decomposed in the form of basis function and the coefficient term. Then one can easily reconstruct the sparse coefficient of minimum number of non-zero coefficient by random by $l_{1}$ minimization. The code and carrier tracking loop of the software GPS receiver has to be synchronized if and only if the lock is achieved. Due to multipath error, the code loop error may be varied more than 1 chip delay and the carrier loop (Costas) is also intercoupled with this, so error may be introduced in the carrier tracking loop also hence, both the tracking errors should be carefully minimized to certain extent to achieve the lock.

The objective function $(\mathrm{J})$ and the 2000 samples of the recovered GPS signal after ISTA algorithm is plotted in Figures 7 and 8 respectively. The recovered signal is further given to the acquisition stage to find the visible satellites (SVN's) and allocate those SVN to initiate the tracking stage. The code and carrier tracking error is observed after recovering the GPS signal using MM method. The significant improvement in carrier tracking is achieved within 50 msec

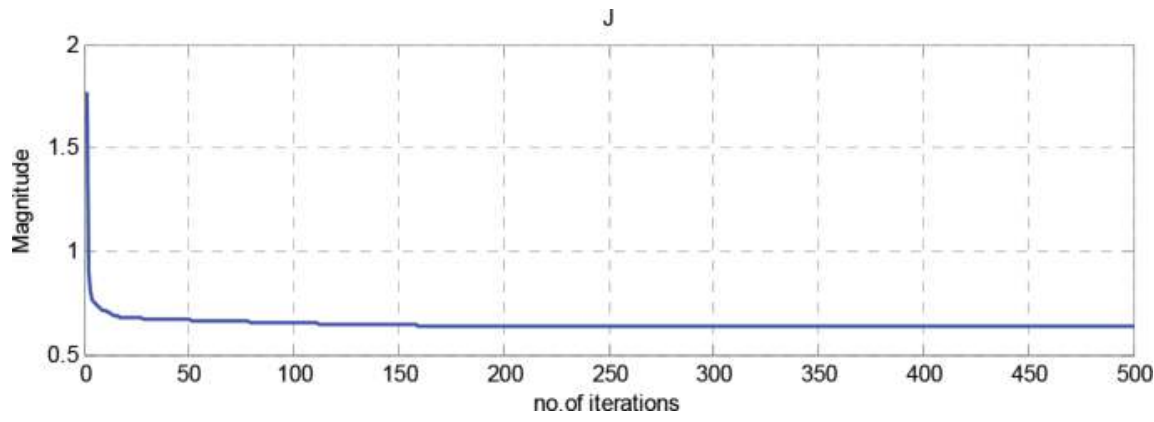

Figure 7. Objective function. 


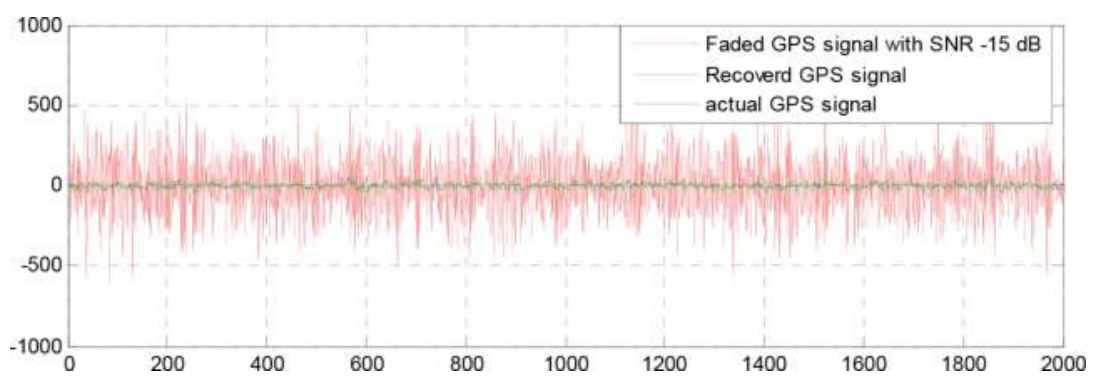

Figure 8. Recovered GPS signal through ISTA algorithm.

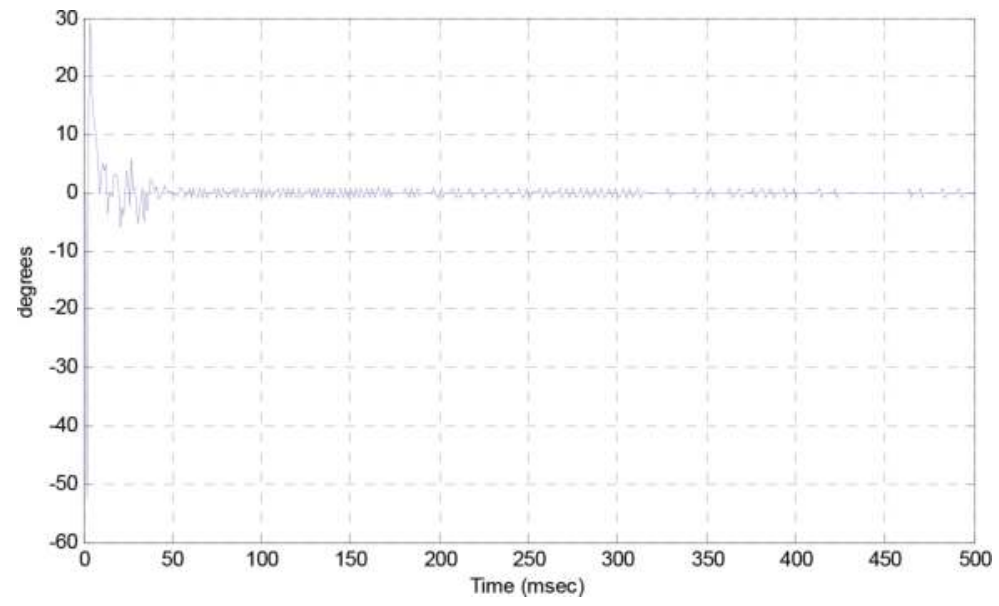

Figure 9. Carrier loop tracking error for SVN-12.

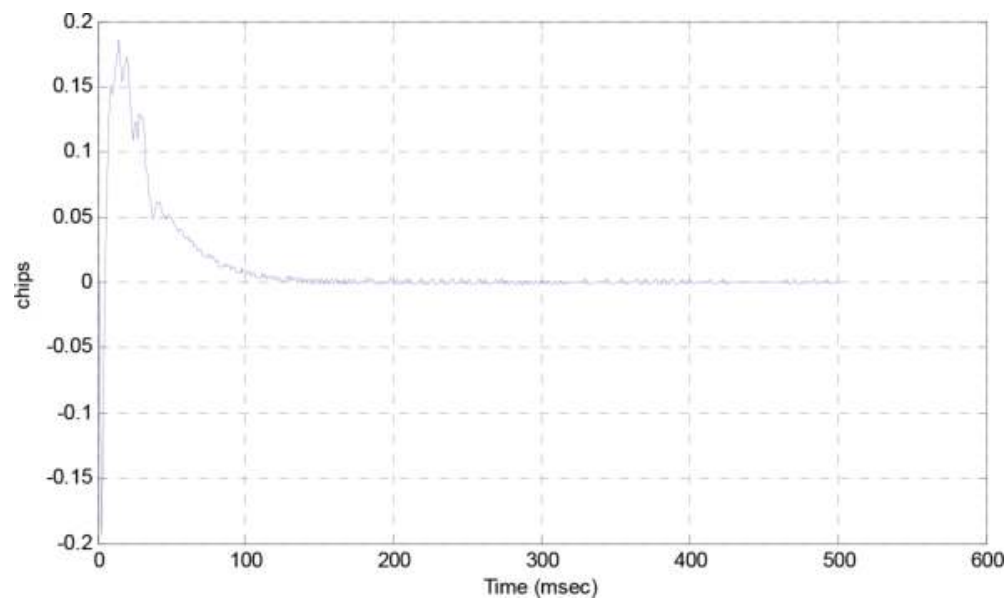

Figure 10. Code loop tracking error for SVN-12. 
period where as in the case of code tracking, error is settled down quickly within $100 \mathrm{msec}$ of GPS data as shown in Figures 9 and 10. The lock has been achieved with 0.5 chips spacing of early, late and prompt code replicas, hence the navigation data can easily be demodulated and the pseudorange is calculated for each satellite.

\section{Conclusion}

In this Chapter, various techniques have been discussed to nullify the effect of the multipath, we have provided an in depth review of existing multipath mitigation techniques. These techniques were classified in categories according to the involved process before and after correlation with the C/A code. Compressive sensing is a promoting tool for the next generation communication systems. However, it still faces a number of challenges in the real time implementation. In multipath applications, compressive sensing exploits the GPS signal need to be converted to a sparse equivalent structure then the channel impulse response of the filter is determined from the convolution matrix. For reconstruction, the challenge resides in how to separate the LOS signal from composite signals in multi-channel environments, where the channel powers and behaviors evolve over time. A comparison of several compressive techniques was given and discussed. The sparse recovery of the signal is obtained from unconstrained optimization algorithms.

\section{Author details}

Ganapathy Arul Elango*, B. Senthil Kumar, Ch.V.M.S.N. Pavan Kumar and C. Venkatramanan *Address all correspondence to: arulelango2012@gmail.com

Department of Electronics and Communication Engineering, Sree Vidyanikethan Engineering College, Tirupathi, India

\section{References}

[1] Michel SB, Van Dierendonck AJ. GPS receiver architecture and measurements. Proceedings of the IEEE. 1999;87:48-64

[2] Borre K, Akos DM, Bertelsen N, Rinder P, Jensen SH. A software defined GPS and Galileo receiver. New York: Birkhäuser Bostonin, Springer Science \& Business Media; 2007. ISBN10 978-0-8176-4390-4

[3] Wildemeersch M. Fortuny-Guasch J. Radio Frequency Interference Impact Assessment on Global Navigation Satellite Systems. Security Technology Assessment Unit, EC Joint Research Centre. In: EUR 24242 EN. 2010 
[4] Arul Elango G, Sudha GF. Design of complete software GPS signal simulator with low complexity and precise multipath channel model. Journal of Electrical Systems and Information Technology. 2016;3(2):161-180

[5] Raju PLN. Fundamentals of GPS, satellite remote sensing and GIS applications in agricultural meteorology. In: Proceedings of a Training Workshop; 7-11 July 2003; Dehradun. pp. 121-150

[6] Tranquilla JM, Carr JP, Al-Rizzo H. Analysis of a choke ring ground plane for multipath control in global positioning system (GPS) applications. IEEE Transactions on Antennas and Propagation; 42(7):905-991

[7] Vagle N, Broumandan A, Jafarnia-Jahromi A, Lachapelle G. Mitigation using antenna arrays. The Journal of Global Positioning Systems. 2016;14:4

[8] Townsend BR, Fenton PC, Van Dierendonck KJ, van Nee DJR. L1 carrier phase multipath error reduction using MEDLL technology. In: Proceedings of ion GPS. Vol. 8. Institute of Navigation; 1995. pp. 1539-1544

[9] Betz JW, Kolodziejski KR. Extended theory of early-late code tracking for a bandlimited GPS receiver. Navigation, Journal of the Institute of Navigation. 2000;47(3):211-226

[10] Benachenhou K, Sari E, Hammadouche M. Multipath mitigation in Gps/Galileo receivers with different signal processing techniques. In: Proceedings of 5th International Conference: Sciences of Electronic Technologies of Information and Telecommunications; March 22-26; Tunisia; 2009. pp. 1-8

[11] Yedukondalu K, Sarma AD, Kumar A, Satyanarayana K. Spectral analysis and mitigation of GPS multipath error using digital filtering for static applications. IETE Journal of Research. 2013;59(2)

[12] Yedukondalu K, Sarma AD, Kumar A. Mitigation of GPS multipath error using recursive least squares adaptive filtering. IEEE Asia Pacific Conference on Circuits and Systems. Kuala Lumpur, Malaysia, 2010:104-107

[13] Bertozzi T, Le Ruyet D, Panazio C, Thien HV. Channel tracking using particle filtering in unresolvable multipath environments. EURASIP Journal on Advances in Signal Processing; 2004(15):705208

[14] Mosavi MR, Azarbad MR. Multipath error mitigation based on wavelet transform in L1 GPS receivers for kinematic applications. AEU - International Journal of Electronics and Communications. 2013;67(10):875-888

[15] Broumandan A. Lin T. Performance of GNSS time of arrival estimation techniques in multipath environments. In: Proceedings of ION GNSS08. Savannah, GA; September; 2008:632-643

[16] Cheng L, Jie C, Gang X. Model and simulation of multipath error in DLL for GPS receiver. Chinese Journal of Electronics, 3. July, 2014;23 
[17] Sleewaegen JM, Boon F. Mitigating short-delay multipath: A promising new technique. In: Proceedings of the International Technical Meeting of the Satellite Division of the Institute of Navigation (ION GPS '01); September 2001; Salt Lake City, Utah, USA. 2001; pp. 204-213

[18] Yedukondalu, A. D. Sarma, And V. Satya Srinivas. Estimation and mitigation of GPS multipath interference using adaptive filtering. Progress in Electromagnetics Research M. 2011;21:133-148

[19] Dragunas K, Borre K. Multipath mitigation based on deconvolution. Journal of Global Positioning Systems. 2011;10(1):79-88

[20] Dragunas K. Indoor multipath mitigation. In: International Conference on Indoor Positioning and Indoor Navigation; September, 2010. pp. 578-584

[21] Sokhandan N, Broumandan A, Dehghanian V, Lachapelle G. GNSS multipath error reduction in harsh environments. In: ION proceedings of GNSS 2011; Portland; September; 2011: 20-23

[22] Kumar R, Lau K. Deconvolution approach to carrier and code multipath error elimination in high precision GPS. In: Proceedings of the 1996 National Technical Meeting of the Institute of Navigation. Santa Monica, CA; 1996:729-737

[23] Xiang F, Liao G, Zeng C, Wang W. A multipath mitigation discriminator for GPS receiver. International Journal of Electronics and Communications. October, 2013;67(10):839-847

[24] Tian Y, Li Y, Lin H, Ma H. A sparse NMF-SU for seismic random noise attenuation. IEEE Geosciences and Remote Sensing Letters. May, 2013;10(3)

[25] Bostan E, Kamilov US. Sparse stochastic processes and discretization of linear inverse problems. IEEE Transactions on Image Processing. July, 2013;22(7):2699-2710 
